\title{
On games without approximate equilibria
}

\author{
Yehuda John Levy ${ }^{1}$ (D)
}

Accepted: 26 August 2020 / Published online: 7 September 2020

(c) The Author(s) 2020

\begin{abstract}
This note shows that the work by Simon and Tomkowicz (Israel J Math 227(1):215231,2018 ) answers another outstanding open question in game theory in addition to the non-existence of approximate Harsányi equilibrium in Bayesian games: it shows that strategic form games with bounded and separately continuous payoffs need not possess approximate equilibria.
\end{abstract}

Keywords Approximate equilibrium · Bayesian games · Discontinuous games

A strategic form game, with bounded and separately continuous payoffs, is a triple $\left\langle N,\left(X_{i}\right)_{i \in N},\left(u_{i}\right)_{i \in N}\right\rangle$, where $N$ a finite collection of players, and for each $i \in N$, $X_{i}$ is a compact convex set in some locally convex topological vector space, and $u_{i}: \prod_{i \in N} X_{i} \rightarrow \mathbb{R}$ is a bounded function which is affine in $X_{i}$ and which is, for each $j \in N$, continuous in $X_{j}$ when other components are held constant. A particular case of such games is when $X_{i}$ is the set of probability measures $\Delta\left(A_{i}\right)$ over a compact set $A_{i}$ in some metric space endowed with the topology of weak convergence, and $u_{i}$ is the multi-linear extension of some bounded separately continuous payoff function on $\prod_{i} A_{i}$, known as the mixed extension (to this extent, $\prod_{i} A_{i}$ embeds naturally into $\prod_{i} X_{i}=\prod_{i} \Delta\left(A_{i}\right)$.)

In the two-player zero-sum case, Sion's minmax theorem, Sion (1958), shows that the separate continuity is sufficient to guarantee existence of the value; the existence of optimal strategies follows. (Indeed, if $u: X_{1} \times X_{2} \rightarrow \mathbb{R}$ is separately continuous, $x \rightarrow \min _{y \in Y} u(x, y)$ is upper-semicontinuous and hence obtains a maximum at an optimal strategy of the maximizer, i.e. of Player 1; and similarly for Player 2.) In the non-zero-sum case, however, an equilibrium need not exist; Vieille (1996) presents an example of a two-player non-zero-sum game 'on the square', i.e. in which each agent has action space $[0,1]$, the mixed extension of which possesses no equilibrium.

Yehuda John Levy

john.levy@glasgow.ac.uk

1 Adam Smith Business School, University of Glasgow, Glasgow, UK 
It has remained open until now, however, the question of whether such games must possess $\varepsilon$-equilibria for each $\varepsilon>0$; a profile $x=\left(x_{i}\right)_{i \in N}$ is a $\varepsilon$-equilibrium iff

$$
\sup _{y_{i} \in X_{i}} u_{i}\left(y_{i}, x_{-i}\right) \leq u_{i}(x)+\varepsilon, \forall i \in N
$$

(The case $\varepsilon=0$ is simply an equilibrium.) This note shows that the example Simon and Tomkowicz (2018) gives a negative answer to this question.

The Bayesian game model of Simon and Tomkowicz (2018) fits into the following general model of Bayesian games: An underlying state space $\Omega$ with $\sigma$-algebra $\mathcal{F}$ and probability measure $\mu$ is given on $\mathcal{F}$. There are $N$ players, each (for simplicity) with a finite action set $A_{i}$, a bounded measurable payoff function $r_{i}: \Omega \times \prod_{i \in N} A_{i} \rightarrow \mathbb{R}$, and a knowledge $\sigma$-algebra $\mathcal{F}_{i} \subseteq \mathcal{F}$. (This model is similar to the model in Stinchcombe (2011), except there the state space is a product of types spaces for each player, endowed with product $\sigma$-algebra; the general framework recalled here translates easily to this one.) A pure (resp. mixed) strategy $\sigma_{i}$ of Player $i$ is a mapping $\Omega \rightarrow A_{i}$ (resp. $\rightarrow \Delta\left(A_{i}\right)$ ) which is $\mathcal{F}_{i}$-measurable. (A pure strategy can be viewed a mixed strategy which assigns only Dirac measures.) For a profile $\sigma=\left(\sigma_{i}\right)_{i \in N}$ of strategies, the payoff to Player $i$ is given by

$$
u_{i}(\sigma)=\int_{\Omega}\left(\sum_{a=\left(a_{1}, \ldots, a_{n}\right) \in \prod_{i \in N} A_{i}} r_{i}(\omega, a) \prod_{i \in N} \sigma_{i}(\omega)\left[a_{i}\right]\right) \mu(d \omega)
$$

Clearly, two strategy profiles $\sigma=\left(\sigma_{i}\right)_{i \in N}$ and $\tau=\left(\tau_{i}\right)_{i \in N}$ which agree $\mu$-a.e. yield the same payoffs, and hence one may treat the strategy space of Player $i$ to be $X_{i}=L^{\infty}\left((\Omega, \mu), \Delta\left(A_{i}\right)\right)$, the space of $\mu$-measurable functions from $\Omega$ to $\Delta\left(A_{i}\right)$ modulo $\mu$-a.e. equality, endowed with the weak-* topology which makes it a compact metrizable space. (See, e.g., Rudin (2006) for the relevant background.) Furthermore, taking this collection of $\left(X_{i}\right)_{i \in N}$ of action spaces makes the payoff functons $\left(u_{i}\right)$ defined in (2) clearly bounded but also separately continuous; indeed,

$u_{i}\left(\sigma_{i}, \sigma_{-i}\right)=\sum_{a_{i} \in A_{i}} \int_{\Omega} \sigma_{i}(\omega)\left[a_{i}\right] \cdot\left(\sum_{a_{-i} \in \prod_{j \neq i} A_{j}} r_{i}\left(\omega, a_{i}, a_{-i}\right) \prod_{j \neq i} \sigma_{j}(\omega)\left[a_{j}\right]\right) \mu(d \omega)$ and for each $a_{i} \in A_{i}$, the function

$$
\omega \rightarrow\left(\sum_{a_{-i} \in \prod_{j \neq i} A_{j}} r_{i}\left(\omega, a_{i}, a_{-i}\right) \prod_{j \neq i} \sigma_{j}(\omega)\left[a_{j}\right]\right)
$$

is in $L^{1}((\Omega, \mu), \mathbb{R})$. Stinchcombe (2011) discusses, at length, how these functions in general are not jointly continuous in all strategies, and characterizes precisely when discontinuities arise. 
The notions discussed in Simon and Tomkowicz (2018) of Harsányi equilibrium and $\varepsilon$-Harsányi equilibrium are precisely the usual notions of equilibrium and $\varepsilon$ equilibrium in the auxilliary strategic form game described above with actions spaces $\left(L^{\infty}\left((\Omega, \mu), \Delta\left(A_{i}\right)\right)\right)_{i \in N}$ and payoffs defined by (2). Using standard measurable selection theorems, the definition given using (1) is equivalent to the following definition:

Definition 1 Fix a Bayesian game as above, and let $\varepsilon>0$. A strategy profile $\sigma=$ $\left(\sigma^{i}\right)_{i \in I}$, with $\sigma_{i} \in X_{i}:=L^{\infty}\left((\Omega, \mu), \Delta\left(A_{i}\right)\right)$, is an Harsányi $\varepsilon$-equilibrium, if for each $i \in I$,

$$
\int_{\omega \in \Omega}\left[\max _{b^{i} \in A^{i}} r^{i}\left(\omega, b^{i}, \sigma^{-i}(\omega)\right)-r^{i}(\omega, \sigma(\omega))\right] d \mu(\omega) \leq \varepsilon .
$$

where $r^{i}$ has been extended multi-linearly to $\prod_{i=1}^{N} \Delta\left(A^{i}\right)$.

In particular, the example in Simon (2003) of a Bayesian game in which no Harsányi equilibrium exists, as well as the somewhat stronger example in Hellman (2014), gave yet more examples of a strategic form game with bounded separately continuous payoff not posessing equilibrium. However, both these examples can be shown to possess $\varepsilon$-Harsányi equilibria for each $\varepsilon>0$; see Hellman and Levy (2020). The example in Simon and Tomkowicz (2018) of a Bayesian game possessing no $\varepsilon$-Harsányi equilibrium hence gives an example of a strategic form game possessing no $\varepsilon$-equilibrium for $\varepsilon>0$ small enough.

It is to be noted that the example in Simon and Tomkowicz (2018) uses three players (see the discussion on Simon and Tomkowicz 2018, p. 230). It is still an open question as to whether two-player bounded separately continuous strategic form games must possess $\varepsilon$-equilibria.

Finally, it is noted:

Proposition 1 The mixed extension of an N-player bounded separately continuous strategic form game, with compact metric action spaces, in which all but at most one player have jointly continuous payoff functions, possesses an $\varepsilon$-equilibrium for each $\varepsilon>0$.

Indeed, in the 2-player game presented in Vieille (1996), the payoff of Player 1 is a quadratic form in two variables, and hence $\varepsilon$-equilibria exist.

Proof Let $\varepsilon>0$. W.l.o.g, $u_{j}(\cdot)$ is jointly continuous in all actions, i.e. continuous on $\prod_{i=1}^{N} X_{i}$, for each $j=1, \ldots, N-1$. For each $j=1, \ldots, N-1$, let $X_{j}^{\prime} \subseteq X_{j}$ be finite, such that for each $x_{j} \in X_{j}$ there is $x_{j}^{\prime} \in X_{j}^{\prime}$ s.t. for all $x_{-j} \in \prod_{i \neq j} X_{i}$, $\left|u_{j}\left(x_{j}, x_{-j}\right)-u_{j}\left(x_{j}^{\prime}, x_{-j}\right)\right|<\varepsilon$, i.e., so that the functions $\left(u_{j}\left(x_{j}^{\prime}, \cdot\right)\right)_{x_{j}^{\prime} \in X_{j}^{\prime}}$ are $\varepsilon$-dense w.r.t. to supremum-norm in the set of functions $\left(u_{j}\left(x_{j}, \cdot\right)\right)_{x_{j} \in X_{j}}$. Such $X_{1}^{\prime}, \ldots, X_{N-1}^{\prime}$ exist by the assumed joint continuity of $u_{1}, \ldots, u_{N-1}$ and the compactness of the action spaces.

For $j=1, \ldots, N$, let $v_{j}$ denote the restriction of $u_{j}$ to $\left(\prod_{i=1}^{N-1} X_{i}^{\prime}\right) \times X_{N}$. The $\left(v_{i}\right)_{i=1}^{N}$ are continuous. Let $x^{*}=\left(x_{1}^{*}, \ldots, x_{N}^{*}\right)$ be an equilibrium of the mixed extension of $\left(v_{i}\right)_{i=1}^{N}$. Clearly, in the original game $\left(u_{j}\right)_{j=1}^{N}$, Player $N$ has no profitable 
deviation against $\left(x_{1}^{*}, \ldots, x_{N-1}^{*}\right)$ in $X_{N}$, as $u_{N}\left(x_{-N}^{*}, \cdot\right)=v_{N}\left(x_{-N}^{*}, \cdot\right)$. Furtheremore, since $x^{*}$ is an equilibrium under $\left(v_{j}\right)_{j=1}^{N}$, it holds for each $j=1, \ldots, N-1$,

$$
u_{j}\left(x^{*}\right)=v_{j}\left(x^{*}\right)=\max _{x \in X_{j}^{\prime}} v_{j}\left(x, x_{-j}^{*}\right)=\max _{x \in X_{j}^{\prime}} u_{j}\left(x, x_{-j}^{*}\right) \geq \max _{x \in X_{j}} u_{j}\left(x, x_{-j}^{*}\right)-\varepsilon
$$

where the last inequality uses the approximation properties of $X_{j}^{\prime}$.

Open Access This article is licensed under a Creative Commons Attribution 4.0 International License, which permits use, sharing, adaptation, distribution and reproduction in any medium or format, as long as you give appropriate credit to the original author(s) and the source, provide a link to the Creative Commons licence, and indicate if changes were made. The images or other third party material in this article are included in the article's Creative Commons licence, unless indicated otherwise in a credit line to the material. If material is not included in the article's Creative Commons licence and your intended use is not permitted by statutory regulation or exceeds the permitted use, you will need to obtain permission directly from the copyright holder. To view a copy of this licence, visit http://creativecommons.org/licenses/by/4.0/.

\section{References}

Hellman Z (2014) A game with no Bayesian approximate equilibria. J Econ Theory 153:138-151

Hellman Z, Levy YJ (2020) Equilibria existence in bayesian games: Climbing the countable borel equivalence relation hierarchy (in preparation)

Rudin W (2006) Functional analysis. Int Ser Pure Appl Math

Simon RS (2003) Games of incomplete information, ergodic theory, and the measurability of equilibria. Israel J Math 138(1):73-92

Simon RS, Tomkowicz G (2018) A Bayesian game without $\varepsilon$-equilibria. Israel J Math 227(1):215-231

Sion M (1958) On general minimax theorems. Pac J Math 8(1):171-176

Stinchcombe MB (2011) Balance and discontinuities in infinite games with type-dependent strategies. J Econ Theory 146(2):656-671

Vieille N (1996) On equilibria on the square. Int J Game Theory 25(2):199-205

Publisher's Note Springer Nature remains neutral with regard to jurisdictional claims in published maps and institutional affiliations. 